\title{
THE BRAUER INDECOMPOSABILITY OF SCOTT MODULES WITH SEMIDIHEDRAL VERTEX
}

\author{
SHIGEO KOSHITANI AND İPEK TUVAY
}

\begin{abstract}
We present a sufficient condition for the $k G$-Scott module with vertex $P$ to remain indecomposable under the Brauer construction for any subgroup $Q$ of $P$ as $k\left[Q C_{G}(Q)\right]$-module, where $k$ is a field of characteristic 2, and $P$ is a semidihedral 2-subgroup of a finite group $G$. This generalizes results for the cases where $P$ is abelian or dihedral. The Brauer indecomposability is defined by R. Kessar, N. Kunugi and N. Mitsuhashi. The motivation of this paper is the fact that the Brauer indecomposability of a $p$ permutation bimodule ( $p$ is a prime) is one of the key steps in order to obtain a splendid stable equivalence of Morita type by making use of the gluing method due to Broué, Rickard, Linckelmann and Rouquier, that then can possibly be lifted to a splendid derived (splendid Morita) equivalence.
\end{abstract}

\section{INTRODUCTION AND NOTATION}

Throughout this paper we denote by $k$ an algebraically closed field of characteristic $p>0$ and by $G$ a finite group. For a $p$-permutation $k G$ module $M$ and a $p$-subgroup $P$ of $G$, the Brauer construction (Brauer quotient) $M(P)$ of $M$ with respect to $P$ plays a very important role. It canonically becomes a $p$-permutation module over $k N_{G}(P)$ (see p.402 in [4]). In their paper [12] Kessar, Kunugi and Mitsuhashi introduce a notion Brauer indecomposability. Namely, $M$ is called Brauer indecomposable if the restriction module $\operatorname{Res}_{Q C_{G}(Q)}^{N_{G}(Q)}[M(Q)]$ is indecomposable or zero as $k\left[Q C_{G}(Q)\right]$-module for any subgroup $Q$ of $P$. Actually in order to get a kind of equivalence between the module categories for the principal block algebras $A$ and $B$ of the group algebras $k G$ and $k H$ respectively (where $H$ is another finite group), e.g. in order to prove Broué's abelian defect group conjecture, we usually first of all have to face a situation such that $A$ and $B$ are stably equivalent of

Date: May 6, 2021.

Keywords: Brauer indecomposability; Scott modules; Brauer construction; Semidihedral group

2010 Mathematics subject classification: 20C20, 20C05, $20 \mathrm{C} 15$. 
Morita type. In order to get the stable equivalence, we often want to check whether the $k(G \times H)$-Scott module $\operatorname{Sc}(G \times H, \Delta P)$ with a vertex $\Delta P:=\{(u, u) \in P \times P\}$ induces a stable equivalence of Morita type between $A$ and $B$, where $P$ is a common Sylow $p$-subgroup of $G$ and $H$ by making use of the gluing method originally due to Broué (see [5]), and also Rickard, Linckelmann and Rouquier. If this is the case, then $\operatorname{Sc}(G \times H, \Delta P)$ have to be Brauer indecomposable. Therefore it should be very important to know whether the $k(G \times H)$-Scott module $\operatorname{Sc}(G \times H, \Delta P)$ is Brauer indecomposable or not. This is the motivation why we have written this paper.

Actually, our main results are the following:

Theorem 1.1. Suppose that a finite group $G$ has a semidihedral 2subgroup $P$. Assume further that the fusion system $\mathcal{F}_{P}(G)$ of $G$ over $P$ is saturated and that $C_{G}(Q)$ is 2-nilpotent for every fully $\mathcal{F}_{P}(G)$ normalized non-trivial subgroup $Q$ of $P$. Then the $\operatorname{Scott}$ module $\operatorname{Sc}(G, P)$ is Brauer indecomposable.

Theorem 1.2. Let $G$ and $G^{\prime}$ be finite groups with a common Sylow 2-subgroup $P$ which is a semidihedral group, and assume that the two fusion systems of $G$ and $G^{\prime}$ over $P$ are the same, namely $\mathcal{F}_{P}(G)=$ $\mathcal{F}_{P}\left(G^{\prime}\right)$. Then the Scott module $\operatorname{Sc}\left(G \times G^{\prime}, \Delta P\right)$ is Brauer indecomposable.

These theorems in a sense generalize [12, 11, 13, 14, and there are results on Brauer indecomposability of Scott modules also in [15, 21].

Notation 1.3. Besides the notations explained above we need the following notations and terminology. In this paper $G$ is always a finite group, and $k$ is an algebraically closed field of characteristic $p>0$.

By a $k G$-module we mean a finitely generated left $k G$-module unless stated otherwise. For a $k G$-module and a $p$-subgroup $P$ of $G$ the Brauer construction (Brauer quotient) $M(P)$ is defined as in $\$ 27$ of [20] or p.402 in [4]. For such $G$ and $P$ we write $\mathcal{F}_{P}(G)$ for the fusion system (fusion category) of $G$ over $P$ as in I.Definition 1.1 of [1]. For two $k G$-modules $M$ and $L$, we write $L \mid M$ if $L$ is (isomorphic to) a direct summand of $M$ as a $k G$-module. For a subgroup $H$ of $G$, a $k G$-module $M$ and a $k H$-module $N$, we write $\operatorname{Res}_{H}^{G}(M)$ for the restriction module $M$ from $G$ to $H$, and $\operatorname{Ind}_{H}^{G}(N)$ for the induced module of $N$ from $H$ to $G$. For a subgroup $H \leq G$ we denote the (Alperin-)Scott $k G$-module with respect to $H$ by $\operatorname{Sc}(G, H)$. By definition, $\operatorname{Sc}(G, H)$ is the unique indecomposable direct summand of $\operatorname{Ind}_{H}^{G}\left(k_{H}\right)$ which contains $k_{G}$ in its top (or equivalently in its socle), where $k_{H}$ and $k_{G}$ are the trivial $k H$ - 
and $k G$-modules, respectively. We refer the reader to $\S 2$ of [4] and in $\S 4.8 .4$ of [18] for further details on Scott modules.

We write $O_{p^{\prime}}(G)$ for the largest normal $p^{\prime}$-subgroup of $G$, and $Z(G)$ for the center of $G$. For $x, y \in G$ we set $x^{y}:=y^{-1} x y$ and ${ }^{y} x:=y x y^{-1}$. Further for a subgroup $H$ of $G$ and $g \in G$ we set ${ }^{g} H:=\left\{{ }^{g} h \mid \forall h \in H\right\}$. For two groups $K$ and $L$ we write $K \rtimes L$ for a semi-direct product of $K$ by $L$ where $K \triangleleft(K \rtimes L)$. For a positive integer $m$, we mean by $C_{m}$ and $S_{m}$ the cyclic group of order $m$ and the symmetric group of degree $m$, respectively. Furthermore $Q_{2^{m}}$ for $m \geq 3$ and $\mathrm{SD}_{2^{m}}$ for $m \geq 4$ are the generalized quaternion group of order $2^{m}$ and the semidihedral group of order $2^{m}$, respectively. In fact more precisely speaking our main character the semidihedral group $\mathrm{SD}_{2^{n}}$ is defined by

$$
\mathrm{SD}_{2^{n}}:=\left\langle x, y \mid x^{2^{n-1}}=y^{2}=1, y^{-1} x y=x^{2^{n-2}-1}\right\rangle \text { where } n \geq 4 .
$$

We fix the notations $x, y$ and $n$ throughout this paper. We also set $z:=x^{2^{n-2}}$, and hence $\langle z\rangle=Z\left(\mathrm{SD}_{2^{n}}\right) \cong C_{2}$, see p.191 of [8].

For the other notations and terminologies, see the books [18], [8] and [1].

The organization of this paper is as follows. In $\S 2$ we give several theorems which have been proved, and also lemmas that are useful of our aim, and already in $\S 2$ we prove our first result Theorem 1.1. In $\S 3$ we prove our second result Theorem 1.2 .

\section{Preliminaries/Lemma}

In this section we list several previous results and lemmas which are useful to prove our main results. The following results of Ishioka and Kunugi will be used for showing Brauer indecomposability of Scott module under consideration.

Theorem 2.1 (Theorem 1.3 of [9]). Assume that $P$ is a p-subgroup of $G$ and $\mathcal{F}_{P}(G)$ is saturated. Then the following assertions are equivalent:

(1) $\operatorname{Sc}(G, P)$ is Brauer indecomposable.

(2) $\operatorname{Res}_{Q C_{G}(Q)}^{N_{G}(Q)} \operatorname{Sc}\left(N_{G}(Q), N_{P}(Q)\right)$ is indecomposable for each fully $\mathcal{F}_{P}(G)$-normalized subgroup $Q$ of $P$.

If these conditions are satisfied, then $(\operatorname{Sc}(G, P))(Q) \cong \operatorname{Sc}\left(N_{G}(Q), N_{P}(Q)\right)$ for each fully $\mathcal{F}_{P}(G)$-normalized subgroup $Q \leq P$.

Theorem 2.2 (Theorem 1.4 of [9]). Assume that $P$ is a p-subgroup of $G$ and $\mathcal{F}_{P}(G)$ is saturated. Let $Q$ be a fully $\mathcal{F}_{P}(G)$-normalized subgroup of $P$. Assume further that there exists a subgroup $H_{Q}$ of $N_{G}(Q)$ satisfying the following conditions: 
(1) $N_{P}(Q)$ is a Sylow p-subgroup of $H_{Q}$ and

(2) $\left|N_{G}(Q): H_{Q}\right|=p^{a}$ for an integer $a \geq 0$.

Then $\operatorname{Res}_{Q C_{G}(Q)}^{N_{G}(Q)} \operatorname{Sc}\left(N_{G}(Q), N_{P}(Q)\right)$ is indecomposable.

The following lemma is essentially a kind of very nice application of Baer-Suzuki Theorem Theorem 3.8.2 of [8]. It surprisingly does play an important role not only for [13 but also for our purpose, though it seems quite elementary. Below, we present a shorter proof than the proof given in Lemma 4.2 of [13].

Lemma 2.3 (Lemma 4.2 of [13]). Let $Q$ be a normal 2-subgroup of $G$ such that $G / Q \cong S_{3}$. Assume further that there is an involution $t \in G \backslash Q$. Then $G$ has a subgroup $H$ such that $t \in H \cong S_{3}$.

Proof. We first claim that there is an involution $y \in G \backslash Q$ which is different from $t$.

Assume that there is no such an element. For any $g \in G$ we have $t^{g} \notin Q$ since $Q \unlhd G$, and hence $t^{g}=t$. So that $t \in Z(G)$. Moreover, $\langle Q, t\rangle=Q\langle t\rangle$ and

$$
Q \unlhd Q\langle t\rangle \unlhd G .
$$

We observe that $Q \neq Q\langle t\rangle$ and that $Q\langle t\rangle \neq G$. Hence $Q\langle t\rangle / Q \unlhd G / Q \cong$ $S_{3}$ implies that $Q\langle t\rangle / Q \cong C_{3}$. However, $Q\langle t\rangle / Q \cong\langle t\rangle /(\langle t\rangle \cap Q)$ is a 2-group, which is a contradiction.

Now, we can write $G=Q K$ where $K=\langle t, y\rangle$. Since $K$ is generated by the two involutions $t$ and $y, K$ is dihedral. Hence there is an element $x \in K$ such that $K=\langle x\rangle \rtimes\langle t\rangle$ and that $x^{t}=x^{-1}$. Since $Q$ is a 2-group and $G / Q \cong S_{3}$, we have 3||$x \mid$. So there exists an element $u$ which is a power of $x$ and is of order 3 . Then $u^{t}=u^{-1}$, so that we can set $H=\langle u, t\rangle \cong S_{3}$.

The following corollary is the corresponding version of Corollary 4.3 in [13] that will be crucial in the proof of our main results. Although the proof is quite similar, we add it for completeness.

Corollary 2.4. Let $P$ be a semidihedral 2-subgroup of $G$, and let $Q$ be a subgroup of $P$. Assume that either $Q \cong C_{2} \times C_{2}$ or $Q \cong Q_{8}$. Assume, moreover, that $C_{G}(Q)$ is 2-nilpotent and $N_{G}(Q) / Q C_{G}(Q) \cong S_{3}$. Then there exists a subgroup $H_{Q}$ of $N_{G}(Q)$ such that $N_{P}(Q)$ is a Sylow 2subgroup of $H_{Q}$ and $\left|N_{G}(Q): H_{Q}\right|$ is a power of 2 (possibly 1 ).

Proof. Since $C_{G}(Q)$ is 2-nilpotent, the group $Q C_{G}(Q)$ is also 2-nilpotent. Let $K:=O_{2^{\prime}}\left(Q C_{G}(Q)\right)$ and let $R \in \operatorname{Syl}_{2}\left(Q C_{G}(Q)\right)$ containing $Q C_{P}(Q)$, so that $Q C_{G}(Q)=K \rtimes R$. Note that since $O_{2^{\prime}}\left(Q C_{G}(Q)\right)=O_{2^{\prime}}\left(C_{G}(Q)\right)$ we have $[K, Q]=1$ and so $K \rtimes Q=K \times Q$. Note that $(K \times Q) \unlhd(K \rtimes$ 
$\left.N_{P}(Q)\right)$. Moreover, since $K$ is a characteristic subgroup of $Q C_{G}(Q)$ and $Q C_{G}(Q) \unlhd N_{G}(Q)$, we have $K \unlhd N_{G}(Q)$, so that $K \times Q \unlhd N_{G}(Q)$.

Let us consider quotients with respect to $L:=K \times Q$ and use the notation $\bar{H}$ for the image of $H \leq G$ under the natural epimorphism $\pi_{L}: N_{G}(Q) \rightarrow N_{G}(Q) / L$. Then $\left(\overline{Q C_{G}(Q)}\right) \cong R / Q$ is a normal 2subgroup of $\overline{N_{G}(Q)}$ and we have an isomorphism

$$
\overline{N_{G}(Q)} /\left(\overline{Q C_{G}(Q)}\right) \cong N_{G}(Q) /\left(Q C_{G}(Q)\right) \cong S_{3}
$$

Note that, $\overline{\left(K \rtimes N_{P}(Q)\right)} \cong N_{P}(Q) / Q \cong C_{2}$ where the latter isomorphism comes from Lemma (viii) of [16]. Note also that,

$$
\overline{\left(K \rtimes N_{P}(Q)\right)} \cap \overline{\left(Q C_{G}(Q)\right)}=\overline{\left(K \rtimes Q C_{P}(Q)\right)}=\overline{1}
$$

where the latter equality is true since $C_{P}(Q)=Z(Q)$ from Lemma (viii) of [16]. So there is an involution $t \in \overline{N_{G}(Q)} \backslash \overline{\left(Q C_{G}(Q)\right)}$ (in fact there is one in $\left.\overline{\left(K \rtimes N_{P}(Q)\right)}\right)$. Hence, by Lemma 2.3 there is a subgroup $H$ of $\overline{N_{G}(Q)}$ such that $t \in H \cong S_{3}$. Set $H_{Q}$ as the preimage of $H$ under $\pi_{L}$. It is easy to see that $H_{Q}$ satisfies the required properties.

Now, we are ready to prove the first main result, namely Theorem 1.1 .

Proof of Theorem 1.1. Set $\mathcal{F}:=\mathcal{F}_{P}(G)$. We want to prove that $M(Q)$ is indecomposable as $Q C_{G}(Q)$-module. It follows from Lemma 4.3(ii) of [12] that $M(P)$ is indecomposable as $P C_{G}(P)$-module. Hence we can assume that $Q \supsetneqq P$. From Lemma (i) of [16], $P$ has exactly three maximal subgroups each of which is either isomorphic to cyclic, dihedral or generalized quaternion. Hence a fully $\mathcal{F}_{P}(G)$-normalized subgroup $Q$ of $P$ is isomorphic to either cyclic or dihedral or generalized quaternion. Thus, from Proposition 3.2 (1) of [7] or Lemma 2.1.(i) of [19], it is easy to see that $\operatorname{Aut}(Q)$ is a 2-group unless $Q$ is isomorphic to $C_{2} \times C_{2}$ or $Q_{8}$.

Suppose that $Q \neq C_{2} \times C_{2}$ or $Q_{8}$. Although the proof for this case is similar to the second paragraph of the proof of Theorem 1.3 of [13], we add the details for the convenience of the reader. Since in this case $\operatorname{Aut}(Q)$ is a 2-group, $N_{G}(Q) / C_{G}(Q)$ is also a 2-group. Thus $N_{G}(Q)$ is 2-nilpotent, since $C_{G}(Q)$ is assumed to be 2-nilpotent. So we can write $N_{G}(Q)=K \rtimes S$ where $K:=O_{2^{\prime}}\left(N_{G}(Q)\right)$ and $S$ is a Sylow 2-subgroup of $N_{G}(Q)$. Since $N_{P}(Q)$ is a 2-subgroup, without loss of generality we can assume that $N_{P}(Q) \leq S$. Let us set $H_{Q}:=K \rtimes N_{P}(Q)$, then $N_{P}(Q)$ is a Sylow 2-subgroup of $H_{Q}$ and $\left|N_{G}(Q): H_{Q}\right|$ is a power of 2 .

Suppose that $Q \cong C_{2} \times C_{2}$ or $Q_{8}$. Then $\operatorname{Out}(Q) \cong S_{3}$ and

$$
C_{2} \cong N_{P}(Q) / Q C_{P}(Q) \leq N_{G}(Q) / Q C_{G}(Q) \hookrightarrow \operatorname{Out}(Q)
$$


where the former isomorphism follows from Lemma (viii) of [16], so that we have two cases. If $N_{G}(Q) / Q C_{G}(Q) \cong C_{2}$, we can argue as in the previous paragraph and get the desired subgroup $H_{Q}$. If $N_{G}(Q) / Q C_{G}(Q) \cong S_{3}$, by Corollary 2.4 we again get $H_{Q}$ with the desired properties.

Therefore for all possible fully $\mathcal{F}_{P}(G)$-normalized $Q$, we find the subgroup $H_{Q}$ satisfying the conditions of Theorem 2.2, so we can conclude that $\operatorname{Sc}(G, P)$ is Brauer indecomposable.

Lemma 2.5. Let $P$ be a subgroup of $G$ which is isomorphic to a semidihedral 2-group such that $\mathcal{F}_{P}(G)$ is saturated. If, furthermore, $Q$ is a fully $\mathcal{F}_{P}(G)$-normalized subgroup of $P$ such that $C_{G}(Q)$ is 2-nilpotent, then $\operatorname{Res}_{Q C_{G}(Q)}^{N_{G}(Q)} \operatorname{Sc}\left(N_{G}(Q), N_{P}(Q)\right)$ is indecomposable, namely, $\operatorname{Res}_{Q C_{G}(Q)}^{N_{G}(Q)}([\operatorname{Sc}(G, P)](Q))$ is indecomposable.

Proof. This follows from the proof of Theorem 1.1 and the final part of Theorem 2.1.

\section{Proof of Theorem 1.2}

The following lemma is used in the proof of our second main result.

Lemma 3.1. Let $P$ be a Sylow 2-subgroup of $G$ which is isomorphic to a semidihedral 2-group. If $Q \leq P$ with $|Q| \geq 8$, then $C_{G}(Q)$ is 2-nilpotent.

Proof. Set $P:=\mathrm{SD}_{2^{n}}:=\left\langle x, y \mid x^{2^{n-1}}=y^{2}=1, x^{y}=x^{2^{n-2}-1}\right\rangle$ where $n \geq 4$ as before. Note that setting $z:=x^{2^{n-2}}$, we have that $Z(P)=$ $\langle z\rangle \cong C_{2}$. If $Q$ contains an element $x^{i} \notin\{1, z\}$, then $C_{G}(Q)$ is 2nilpotent, because from line -3 on page 246 of [2] we know that for such $x^{i}, C_{G}\left(x^{i}\right)$ is 2-nilpotent. So, in order to prove the claim, it is enough to show that if $|Q| \geq 8$, then there is an element $x^{i} \notin\{1, z\}$ which lies in $Q$.

By Lemma (ii) of [16], the elements $x^{i} y$ has order 2 or 4 , when $i$ is even or odd, respectively. So if $Q$ is cyclic, then it must contain $x^{i}$ which has order greater or equal to 8 , hence the claim is true. So, we may assume that $Q$ is generated by two elements. We have two cases for this situation, either $Q=\left\langle x^{i} y, x^{j} y\right\rangle$ or $Q=\left\langle x^{\kappa} y, x^{l}\right\rangle$ where $i, j, \kappa, l$ are integers in the set $\left\{1,2, \ldots, 2^{n-1}\right\}$ with $i \neq j$.

In the first case, $Q$ has the element $a_{i, j}:=\left(x^{i} y\right)\left(x^{j} y\right)=x^{i-j} z^{j}$ inside itself. If both $i$ and $j$ are even, then since $z^{j}=1$ we have that $a_{i, j}=x^{i-j}$. If $x^{i-j} \notin\{1, z\}$, we have the result. Otherwise, since $i \neq j, x^{i-j}$ can not be trivial, hence there is only one possibility left: $x^{i-j}=z$, but this implies that $\left(x^{i} y\right)\left(x^{j} y\right)=\left(x^{j} y\right)\left(x^{i} y\right)=z$, hence 
$Q \cong C_{2} \times C_{2}$ contradicting with the assumption $|Q| \geq 8$. So $x^{i-j}=z$ can not happen. If one of $i$ or $j$ is even and the other one is odd, then $a_{i, j}=x^{i-j} z^{j}$ where $i-j$ is odd. So, in this case $a_{i, j}$ is a nontrivial element in $\langle x\rangle \backslash Z(P)$, whence the result. If both $i$ and $j$ are odd, then $a_{i, j}=x^{i-j} z$. Similarly, if $x^{i-j} \notin\{1, z\}$, we have the result. Otherwise, since $i \neq j$, there is only one possibility left: $x^{i-j}=z$. In this case, $\left(x^{i} y\right)\left(x^{j} y\right)=z . z=1$ so that $x^{i} y=\left(x^{j} y\right)^{-1}$. But this is impossible because if this holds $Q \cong C_{4}$ contradicting our assumption. So $x^{i-j}=z$ can not happen in this case, too.

In the second case, if $x^{l}$ lies outside $Z(P)$, the result is true. So let us assume that $x^{l}=z$ (since $x^{l}$ is non-tirivial by the assumptions), then $Q=\left\langle x^{\kappa} y, z\right\rangle$. If $\kappa$ is even then $x^{\kappa} y$ is an involution and commutes with the central involution $z$, hence $\left\langle x^{\kappa} y, z\right\rangle \cong C_{2} \times C_{2}$. This gives a contradiction with our assumption on $Q$. If $\kappa$ is odd, then $\left(x^{\kappa} y\right)^{2}=z$, so $Q \cong C_{4}$, which is similarly a contradiction with the property of $Q$.

Proof of Theorem 1.2. Set $\mathcal{H}:=G \times G^{\prime}$. Since $P$ is a Sylow 2-subgroup of $G, \mathcal{F}:=\mathcal{F}_{P}(G)$ is a saturated fusion system (see Proposition 1.3 of [3]). Moreover since $\mathcal{F}_{\Delta P}(\mathcal{H}) \cong \mathcal{F}_{P}(G)$, we have that $\mathcal{F}_{\Delta P}(\mathcal{H})$ is saturated.

Let $\Delta Q \leq \Delta P$ be any fully $\mathcal{F}_{\Delta P}(\mathcal{H})$-normalized subgroup of $\Delta P$. Setting $M:=\operatorname{Sc}(\mathcal{H}, \Delta P)$, we claim that $M(\Delta Q)$ is indecomposable as a $\Delta Q C_{\mathcal{H}}(\Delta Q)$-module. Note that $C_{\mathcal{H}}(\Delta Q)=C_{G}(Q) \times C_{G^{\prime}}(Q)$.

Case 1: $|Q| \geq 8$ : If $Q$ is a subgroup of $P$ such that $|Q| \geq$ 8, by Lemma 3.1 both $C_{G}(Q)$ and $C_{G^{\prime}}(Q)$ are 2-nilpotent. Hence, $C_{\mathcal{H}}(\Delta Q)$ is also 2-nilpotent. Therefore, by Lemma 2.5 we have that $\operatorname{Res}_{\Delta Q C_{\mathcal{H}}(\Delta Q)}^{N_{\mathcal{H}}(\Delta Q)} M(\Delta Q)$ or equivalently

$$
\operatorname{Res}_{\Delta Q C_{\mathcal{H}}(\Delta Q)}^{N_{\mathcal{H}}(\Delta Q)} \operatorname{Sc}\left(N_{\mathcal{H}}(\Delta Q), N_{\Delta P}(\Delta Q)\right)
$$

is indecomposable for such $Q$.

Case 2: Either $Q \cong C_{2} \times C_{2}$ or $Q \cong C_{4}$ : Let $Q$ be a fully $\mathcal{F}_{\text {- }}$ normalized subgroup of $P$ such that $Q \cong C_{2} \times C_{2}$. Since by Lemma (viii) of [16] there is only one $P$-conjugacy class of Klein four subgroups of $P$, we can assume $Q:=Z(P) \times\langle y\rangle$. Then, again by Lemma (viii) of [16], $C_{P}(Q)=Q$, and by Proposition 2.5 of [17, $Q$ is fully $\mathcal{F}$ centralized, so that from Lemma 2.10(i) of [17], $Q$ is a Sylow 2-subgroup of $C_{G}(Q)$. Hence the Schur-Zassenhaus Theorem yields that $C_{G}(Q)=$ $Q \times O_{2^{\prime}}\left(C_{G}(Q)\right)$, so that $C_{G}(Q)$ is 2-nilpotent. Similarly, $C_{G^{\prime}}(Q)$ is also 2-nilpotent. Thus, $C_{\mathcal{H}}(\Delta Q)$ is also 2-nilpotent. Hence Lemma 2.5 implies that $\operatorname{Res}_{C_{\mathcal{H}}(\Delta Q)}^{N_{\mathcal{H}}(\Delta Q)}[M(\Delta Q)]$ is indecomposable. 
Now, let $Q$ be a fully $\mathcal{F}$-normalized subgroup of $P$ such that $Q \cong$ $C_{4}$. By Theorem of [16] either all elements of order 4 are $G$-conjugate or there are exactly two $G$-conjugacy classes of elements of order 4 . The same situation holds for $G^{\prime}$-conjugacy classes and hence for $\mathcal{F}$ conjugacy classes. In the first case, $\left\langle x^{2^{n-3}}\right\rangle$ is a fully $\mathcal{F}$-normalized representative of the single $\mathcal{F}$-conjugacy class of cyclic subgroups of order 4 of $P$. In the second case, $\left\langle x^{2^{n-3}}\right\rangle$ and $\langle x y\rangle$ are representatives of fully $\mathcal{F}$-normalized subgroups of two $\mathcal{F}$-conjugacy classses of cyclic subgroups of order 4 of $P$. If $Q=\left\langle x^{2^{n-3}}\right\rangle$, then line -3 on page 246 of [2] implies that both $C_{G}(Q)$ and $C_{G^{\prime}}(Q)$ are 2-nilpotent and hence $C_{\mathcal{H}}(\Delta Q)$ is a 2-nilpotent subgroup of $\mathcal{H}$. Therefore Lemma 2.5 implies that $\operatorname{Res}_{C_{\mathcal{H}}(\Delta Q)}^{N_{\mathcal{H}}(\Delta Q)}[M(\Delta Q)]$ is indecomposable. If $Q=\langle x y\rangle$, then since $Q$ is fully $\mathcal{F}$-normalized, it is fully $\mathcal{F}$-centralized (see Proposition 2.5 of [17]), and hence by Lemma 2.10 (i) of [17], $C_{P}(Q) \in \operatorname{Syl}_{2}\left(C_{G}(Q)\right)$, and similarly $C_{P}(Q) \in \operatorname{Syl}_{2}\left(C_{G^{\prime}}(Q)\right)$. From Lemma (ix) of [16], $C_{P}(Q)=$ $Q$, and hence $C_{P}(Q)$ is a normal abelian Sylow 2-subgroup of both $C_{G}(Q)$ and $C_{G^{\prime}}(Q)$. Hence the Schur-Zassenhaus Theorem implies that $C_{G}(Q)=Q \times O_{2^{\prime}}\left(C_{G}(Q)\right)$ and similarly for $C_{G^{\prime}}(Q)$. In particular, both of them are 2-nilpotent. Therefore $C_{\mathcal{H}}(\Delta Q)$ is 2-nilpotent. Thus by Lemma 2.5, we have that $\operatorname{Res}_{C_{\mathcal{H}}(\Delta Q)}^{N_{\mathcal{H}}(\Delta Q)}[M(\Delta Q)]$ is indecomposable in this case, too.

Case 3: $Q \cong C_{2}$ : Let $Q$ be a subgroup of $P$ which is isomorphic to a cyclic group of order 2. From Theorem in [16], either all involutions in $P$ are $G$-conjugate or there are two $G$-conjugacy classes of involutions of $P$ represented by $z$ and $y$. Similar situation holds for $G^{\prime}$-conjugacy classes and for $\mathcal{F}$-conjugacy classes. Hence, it is enough to prove the desired result for $Q=Z(P)$ and for $Q=\langle y\rangle$, since they are the fully $\mathcal{F}$-normalized elements in their own $\mathcal{F}$-conjugacy classes.

Suppose first that $Q=Z(P)$, then $\Delta P \leq N_{\mathcal{H}}(\Delta Q)$. Suppose also that $M(\Delta Q)=M_{1} \oplus \ldots \oplus M_{r}$ where $r \geq 1$ and $M_{i}$ are indecomposable $N_{\mathcal{H}}(\Delta Q)$-modules. From Theorem 4.8.6 (ii)] of [18] we can set $M_{1}:=\operatorname{Sc}\left(N_{\mathcal{H}}(\Delta Q), \Delta P\right)$. Since $M(\Delta Q) \mid \operatorname{Res}_{N_{\mathcal{H}}(\Delta Q)}^{\mathcal{H}}(M)$, we have that $M_{i} \mid \operatorname{Res}_{N_{\mathcal{H}}(\Delta Q)}^{\mathcal{H}}(M)$ for each $i$. Moreover since $M \mid \operatorname{Ind}_{\Delta P}^{\mathcal{H}}(k)$, by Mackey decomposition we have that for a fixed $i \geq 2$,

$$
M_{i} \mid \bigoplus_{h} \operatorname{Ind}_{N_{\mathcal{H}}(\Delta Q) \cap h_{\Delta P}}^{N_{\mathcal{H}}(\Delta Q)}(k)
$$

where $h$ runs over representatives of the double cosets in $N_{\mathcal{H}}(\Delta Q) \backslash \mathcal{H} / \Delta P$ which satisfies $\Delta Q \leq{ }^{h} \Delta P$ by 1.4 of [4]. Hence a vertex $\Delta R$ of $M_{i}$ lies in $N_{h_{\Delta P}}(\Delta Q)=N_{\mathcal{H}}(\Delta Q) \cap{ }^{h} \Delta P$ for some $h$. Note that for such $h$, we 
have that

$$
\Delta R \leq N_{h_{\Delta P}}(\Delta Q) \leq_{N_{\mathcal{H}}(\Delta Q)} \Delta P
$$

by Lemma 3.2 of $[9]$. So, we have that $M_{1}(\Delta R) \neq 0$. On the other hand, by applying Burry-Carlson-Puig's Theorem for $\Delta Q$ (see Theorem 4.4 .6 (ii)] of [18]), each $M_{i}$ has vertex not equal to $\Delta Q$. Hence $\Delta Q$ is a proper normal subgroup of $\Delta R$ and so

$$
M_{1}(\Delta R) \oplus M_{i}(\Delta R) \mid(M(\Delta Q))(\Delta R) \cong M(\Delta R)
$$

as $N_{\mathcal{H}}(\Delta R) \cap N_{\mathcal{H}}(\Delta Q)$-modules, but $C_{\mathcal{H}}(\Delta R) \leq N_{\mathcal{H}}(\Delta R) \cap N_{\mathcal{H}}(\Delta Q)$, so the isomorphism above restricts to as $C_{\mathcal{H}}(\Delta R)$-modules which contradicts with the fact that $M(\Delta R)$ is indecomposable as a $C_{\mathcal{H}}(\Delta R)$ module by Case 1 and Case 2. Therefore, $r=1$ and $M(\Delta Q)$ is indecomposable as a $C_{\mathcal{H}}(\Delta Q)$-module since $C_{\mathcal{H}}(\Delta Q)=N_{\mathcal{H}}(\Delta Q)$.

Suppose now that $Q=\langle y\rangle$, and set $S:=C_{P}(Q)$. Then, $S=$ $C_{P}(Q)=N_{P}(Q)=\langle y, z\rangle \cong C_{2} \times C_{2}$. Assume similarly that $M(\Delta Q)=$ $M_{1} \oplus \ldots \oplus M_{r}$ where $M_{i}$ are indecomposable $N_{\mathcal{H}}(\Delta Q)$-modules. By Lemma 3.1 of [9] and Theorem 1.7 of [10] we can set $M_{1}:=\operatorname{Sc}\left(N_{\mathcal{H}}(\Delta Q), \Delta S\right)$. Since $M \mid \operatorname{Ind}_{\Delta P}^{\mathcal{H}}(k)$, we have that

$$
M(\Delta Q) \mid \bigoplus_{h} \operatorname{Ind}_{N_{\mathcal{H}}(\Delta Q) \cap{ }^{h} \Delta P}^{N_{\mathcal{H}}(\Delta Q)}(k)
$$

where $h$ runs over representatives of the double cosets in $N_{\mathcal{H}}(\Delta Q) \backslash \mathcal{H} / \Delta P$ which satisfies $\Delta Q \leq{ }^{h} \Delta P$ by 1.4 of [4]. Hence,

$$
M_{i} \mid \bigoplus_{h} \operatorname{Ind}_{N_{\mathcal{H}}(\Delta Q) \cap{ }^{h} \Delta P}^{N_{\mathcal{H}}(\Delta Q)}(k)
$$

for each $i$, where $h$ runs through the same set. Hence, for a fixed $i$, a vertex of $M_{i}$ is contained in $N_{h_{\Delta P}}(\Delta Q)$. Note that for such $h$, we have that

$$
N_{h_{\Delta P}}(\Delta Q) \leq_{N_{\mathcal{H}}(\Delta Q)} \Delta S
$$

by Lemma 3.2 of [9]. So for a fixed $i$, any vertex of $M_{i}$ is contained in $\Delta S$. On the other hand, by Lemma 2.1 of [9] and Burry-Carlson-Puig's Theorem (see Theorem 4.4 .6 (ii) in [18]), a vertex of $M_{i}$ contains $\Delta Q$ properly. Hence each $M_{i}$ has a vertex $\Delta S$ (Note that $|S: Q|=2$ ). This implies that $M_{i}(\Delta S) \neq 0$ by (27.7) Corollary in [20]. Therefore, since $\Delta Q$ is normal in $\Delta S$,

$$
M(\Delta S) \cong(M(\Delta Q))(\Delta S)=M_{1}(\Delta S) \oplus \ldots \oplus M_{r}(\Delta S)
$$

as $N_{\mathcal{H}}(\Delta S) \cap N_{\mathcal{H}}(\Delta Q)$-modules. Since $C_{\mathcal{H}}(\Delta S) \leq N_{\mathcal{H}}(\Delta S) \cap N_{\mathcal{H}}(\Delta Q)$ and since we have already proved in Case 2 that $M(\Delta S)$ is indecomposable as a $C_{\mathcal{H}}(\Delta S)$-module (recall that $S \cong C_{2} \times C_{2}$ ), we have $r=1$, so $M(\Delta Q)$ is an indecomposable $C_{\mathcal{H}}(\Delta Q)$-module. 
Acknowledgements. The first author was supported by the Japan Society for Promotion of Science (JSPS), Grant-in-Aid for Scientific Research (C)19K03416, 2019-2021. The second author was supported by Mimar Sinan Fine Arts University Scientific Research Unit with project number 2019-28. The authors thank Caroline Lassueur for useful information on [7].

\section{REFERENCES}

[1] M. Aschbacher, R. Kessar, B. Oliver, Fusion Systems in Algebra and Topology, London Math. Soc. Lecture Note Series vol.391, Cambridge University Press, Cambridge, 2011.

[2] R. Brauer, Some applications of the theory of blocks of characters of finite groups III, J. Algebra 3 (1966), 225-255.

[3] C. Broto, R. Levi, B. Oliver, The homotopy theory of fusion systems, J. Amer. Math. Soc. 16 (2003), 779-856.

[4] M. Broué, On Scott modules and $p$-permutation modules: an approach through the Brauer morphism, Proc. Amer. Math. Soc. 93 (1985), 401-408.

[5] M. Broué, Equivalences of blocks of group algebras, In Finite-dimensional Algebras and Related Topics (Ottawa 1992), Eds. V. Dlab and L.L. Scott, Kluwer Academic Pub., Derdrecht, 1994, pp.1-26.

[6] M. Broué, L. Puig, Characters and local structure in G-algebras, J. Algebra 63 (1980), 306-317.

[7] D. Craven, A. Glesser, Fusion systems on small p-groups, Trans. Amer. Math. Soc. 364 (2012), 5945-5967.

[8] D. Gorenstein, Finite Groups, Harper and Row, New York, 1968.

[9] H. Ishioka, N. Kunugi, Brauer indecomposability of Scott modules, J. Algebra 470 (2017), 441-449.

[10] H. Kawai, On indecomposable modules and blocks, Osaka J. Math. 23 (1986), 201-205.

[11] R. Kessar, S. Koshitani, M. Linckelmann, On the Brauer indecomposability of Scott modules, Quarterly J. Math. 66 (2015), 895-903.

[12] R. Kessar, N. Kunugi, N. Mitsuhashi, On saturated fusion systems and Brauer indecomposability of Scott modules, J. Algebra 340 (2011), 90-103.

[13] S. Koshitani, C. Lassueur, Splendid Morita equivalences for principal 2-blocks with dihedral defect groups, Math. Z. 294 (2020) 639-666.

[14] S. Koshitani, C. Lassueur, Splendid Morita equivalences for principal 2-blocks with generalised quaternion defect groups, J. Algebra 558 (2020) 523-533.

[15] S. Koshitani, İ. Tuvay, The Brauer indecomposability of Scott modules for the quadratic group Qd(p), Algebr. Represent. Theory 22 (2019) 1387-1397.

[16] T. Kwon, K. Lee, I. Cho, S. Park, On finite groups with quasi-dihedral Sylow 2-groups, J. Korean Math. Soc. 17 (1980), 91-97.

[17] M. Linckelmann, Introduction to fusion systems, In: Group Representation Theory, Edited by EPFL Press, 79-113, Lausanne, 2007. 
[18] H. Nagao, Y. Tsushima, Representations of Finite Groups, Academic Press, New York, 1989.

[19] J.B. Olsson, On 2-blocks with quaternion and quasidihedral defect groups, J. Algebra 36 (1975), 212-241.

[20] J. Thévenaz, G-Algebras and Modular Representation Theory, Oxford Sci. Publication, Clarendon, 1995.

[21] İ. Tuvay, On Brauer indecomposability of Scott modules of Park-type groups, J. Group Theory 17 (2014), 1071-1079.

Center for Frontier Science, Chiba University, 1-33 Yayoi-cho, InageKU, CHIBA 263-8522, JAPAN.

Email address: koshitan@math.s.chiba-u.ac.jp

Mimar Sinan Fine Arts University, Department of Mathematics, 34380, Bomonti, Şişli, Istanbul, Turkey

Email address: ipek.tuvay@msgsu.edu.tr 